\title{
Quantitative Longitudinal Antibody Monitoring in a COVID-19 Outpatient Case Series: Relations of IgM/IgG Dynamics to Anosmia and Ageusia
}

Nathalie Gil ${ }^{1}$, Jenny Heneriz ${ }^{1}$, Dennis Diaz ${ }^{1}$, Mike Tomas ${ }^{1}$, Gualberto Ruaño ${ }^{2}$, and Raul Cruz $^{1}$

${ }^{1}$ Fastlabs

${ }^{2}$ Hartford Hospital

April 15, 2021

\begin{abstract}
A case series of 22 patients with confirmed SARS-CoV-2 infection and Corona Virus Disease 2019 (COVID-19) symptoms was followed in a primary care clinic. We provided quantitative and longitudinal profiling for SARS-CoV-2 IgM and IgG with a point of care device. Half the patients had a history of anosmia or ageusia. IgM and IgG responses were highly heterogeneous quantitatively and temporally. We determined that clinical symptomatology of chemosensory loss correlated with lesser but sustained titers of IgM and IgG whereas normal chemosensation correlated with transiently higher but rapidly declining antibody levels.
\end{abstract}

\section{Hosted file}

Quantitative Longitudinal Antibody Monitoring numbered.pdf available at https://authorea.com/ users/407436/articles/517756-quantitative-longitudinal-antibody-monitoring-in-a-covid19-outpatient-case-series-relations-of-igm-igg-dynamics-to-anosmia-and-ageusia 